\title{
Disorder in order: Localization without randomness in a cold-atom system
}

\author{
Félix Rose $\odot^{1,2,3, *}$ and Richard Schmidt $\oplus^{1,2}$ \\ ${ }^{1}$ Max Planck Institute of Quantum Optics, Hans-Kopfermann-Straße 1, 85748 Garching, Germany \\ ${ }^{2}$ Munich Center for Quantum Science and Technology, Schellingstraße 4, 80799 Munich, Germany \\ ${ }^{3}$ Physik Department, Technische Universität München, James-Franck-Strasse 1, 85748 Garching, Germany
}

(Received 22 July 2021; revised 28 December 2021; accepted 10 January 2022; published 28 January 2022)

\begin{abstract}
We present a mapping between the Edwards model of disorder describing the motion of a single particle subject to randomly positioned static scatterers and the Bose polaron problem of a light quantum impurity interacting with a Bose-Einstein condensate (BEC) of heavy atoms. The mapping offers an experimental setting to investigate the physics of Anderson localization where, by exploiting the quantum nature of the BEC, the time evolution of the quantum impurity emulates the disorder-averaged dynamics of the Edwards model. Valid in any space dimension, the mapping can be extended to include interacting particles, arbitrary disorder, or confinement and can be generalized to study many-body localization. Moreover, the corresponding exactly solvable disorder model offers means to benchmark variational approaches used to study polaron physics. Here we illustrate the mapping by focusing on the case of an impurity interacting with a one-dimensional BEC through a contact interaction. While a simple wave function based on the expansion in the number of bath excitations misses the localization physics entirely, a coherent state Ansatz combined with a canonical transformation captures the physics of disorder and Anderson localization.
\end{abstract}

DOI: 10.1103/PhysRevA.105.013324

\section{INTRODUCTION}

Coupling a particle to the collective excitation of a system with many degrees of freedom can radically alter the particle's properties. While this paradigm was first proposed by Landau and Pekar to describe how the interaction between electrons and lattice phonons gives rise to quasiparticles named polarons [1], it has been extended to give insight into numerous systems [2,3], including ${ }^{3} \mathrm{He}-{ }^{4} \mathrm{He}$ mixtures [4], semiconductors [5], and high-temperature superconductors [6]. Although described by simple models such as the Fröhlich Hamiltonian and in spite of intensive efforts [7-22], a comprehensive solution of the polaron problem still escapes theory. The advent of ultracold atoms allows the realization of polaron models with high tunability and introduced means to probe such models, as evidenced by the recent observation of the Bose polaron spectral function in impurity-boson mixtures [23-27].

In this article we bring together the seemingly disconnected fields of polarons and disorder. Following Anderson's realization that quantum interference can hinder the diffusion of a particle to the point that it becomes localized [28], the interplay of disorder and quantum physics has been extensively studied, revealing intricate phenomena such as magnetoresistance [29,30], coherent backscattering [31-33], and many-body localization [34-37]. While

\footnotetext{
*felix.rose@m4x.org
}

Published by the American Physical Society under the terms of the Creative Commons Attribution 4.0 International license. Further distribution of this work must maintain attribution to the author(s) and the published article's title, journal citation, and DOI. Open access publication funded by the Max Planck Society.
Anderson localization was first observed in wave systems $[38,39]$, the development of cold-atom physics allowed achievement of the localization of matter waves $[40,41]$ and realization of the quantum kicked rotor model [42,43], which can be mapped onto the Anderson model of disorder [44].

Here we explore an alternative way to study the physics of disorder by establishing a mapping between the disorderaveraged motion of a single particle evolving through a random disorder potential and that of an impurity immersed in a disorder-free Bose-Einstein condensate (BEC). This mapping, illustrated in Fig. 1, can experimentally be realized using a mass-imbalanced mixture of light impurities immersed in a bath of heavy bosons and provides a theoretical tool to include disorder effects in many-body approaches.

Indeed, the theoretical descriptions of both disorder and Bose polarons face challenges of different origins. While the rich physics of the Bose polaron problem arises from hard-to-capture many-body effects, performing the disorder average for even single-particle models is challenging. As such, a solution of the polaron problem can give insight into the corresponding disorder model and conversely the disorder model can serve as an exactly solvable benchmark for polaron theories. To demonstrate this connection we show how a variational method applied to the study of impurity models can reproduce the exact short-time solution of the corresponding disorder model and discuss implications of the mapping for studies of polaron and disorder physics.

\section{MAPPING}

Many aspects of disorder physics such as Anderson localization are universal. They may depend on dimension and symmetries but not on specific details of the Hamiltonian, 


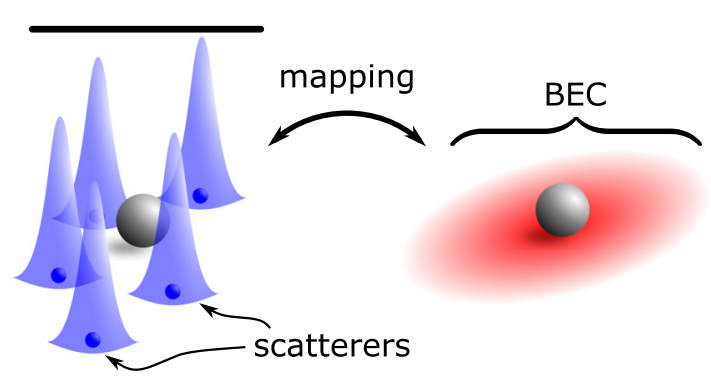

FIG. 1. The disorder-averaged evolution, represented by the overline, of a particle subject to a random scattering potential (left) is mapped onto the time evolution of an impurity immersed in a homogeneous disorder-free BEC (right).

leaving freedom as to which model of disorder one studies. We consider the Edwards model $[45,46]$ that describes the evolution of a single particle through a medium of $N$ randomly positioned static scatterers given by the Hamiltonian

$$
\hat{H}_{\mathrm{Ed}}^{\left\{\mathbf{r}_{i}\right\}}=\frac{\hat{\mathbf{p}}^{2}}{2 m_{\mathrm{I}}}+V(\hat{\mathbf{r}}), \quad V(\hat{\mathbf{r}})=\sum_{i=1}^{N} v\left(\hat{\mathbf{r}}-\mathbf{r}_{i}\right) .
$$

Here $\hat{\mathbf{r}}$ and $\hat{\mathbf{p}}$ are the position and momentum operators of the particle and $v\left(\hat{\mathbf{r}}-\mathbf{r}_{i}\right)$ is the potential created by a scatterer at site $\mathbf{r}_{i}$. In one dimension and with contact interactions, this model is known as the random Kronig-Penney model (RKPM) [47,48]. The randomness comes from the positions $\mathbf{r}_{i}$ of the scatterers, distributed, e.g., uniformly in a volume $\Omega$. Denoting by $\langle\hat{o}(t)\rangle_{\left\{\mathbf{r}_{i}\right\}}$ the expectation value of an observable $\hat{o}$ at time $t$ for a given realization of disorder $\left\{\mathbf{r}_{i}\right\}$, its disorder average is

$$
\overline{\langle\hat{o}(t)\rangle}=\int_{\left\{\mathbf{r}_{i}\right\}}\langle\hat{o}(t)\rangle_{\left\{\mathbf{r}_{i}\right\}},
$$

where $\int_{\left\{\mathbf{r}_{i}\right\}}=\Omega^{-N} \int d^{d} \mathbf{r}_{1} \cdots d^{d} \mathbf{r}_{N}$ is the normalized integral over all possible scatterer positions $\mathbf{r}_{i}$.

We now show that for any observable $\hat{o}$ the disorder average (2) can be computed by means of a disorder-free polaron model. In this model one considers a system where a mobile impurity of position $\hat{\mathbf{r}}$ and momentum $\hat{\mathbf{p}}$ is immersed in a bosonic bath, described by the Hamiltonian

$$
\hat{H}=\frac{\hat{\mathbf{p}}^{2}}{2 m_{\mathrm{I}}}+\sum_{\mathbf{k}} \omega_{\mathbf{k}} \hat{b}_{\mathbf{k}}^{\dagger} \hat{b}_{\mathbf{k}}+\int_{\mathbf{r}^{\prime}} v\left(\hat{\mathbf{r}}-\mathbf{r}^{\prime}\right) \hat{b}_{\mathbf{r}^{\prime}}^{\dagger} \hat{b}_{\mathbf{r}^{\prime}}
$$

where $\omega_{\mathbf{k}}=\mathbf{k}^{2} / 2 m_{\mathrm{B}}$ describes the dispersion relation of bosons at momentum $\mathbf{k}$ annihilated (created) by the operator $\hat{b}_{\mathbf{k}}\left(\hat{b}_{\mathbf{k}}^{\dagger}\right)$, and $m_{\mathrm{I}}$ and $m_{\mathrm{B}}$ are the masses of the impurity and the bosons, respectively. Both species interact with the densitydensity interaction $v(\mathbf{r})$.

To establish the mapping, we quench the system by preparing the impurity in a given wave packet $|\psi\rangle$ and the bosons in a Bose-Einstein condensate of $N$ noninteracting particles,

$$
|\mathrm{BEC}\rangle=\frac{\left(\hat{b}_{\mathbf{k}=0}^{\dagger}\right)^{N}}{\sqrt{N !}}|0\rangle=\int_{\left\{\mathbf{r}_{i}\right\}}\left|\left\{\mathbf{r}_{i}\right\}\right\rangle,
$$

TABLE I. Correspondence between the heavy Bose polaron model and the Edwards model of disorder.

\begin{tabular}{lc}
\hline \hline Polaron model & Disorder model \\
\hline Impurity & Particle \\
Heavy boson & Static scatterer \\
Interspecies interaction & Scattering potential \\
$N$-boson state $\left|\left\{\mathbf{r}_{i}\right\}\right\rangle$ & Disorder configuration $\left\{\mathbf{r}_{i}\right\}$ \\
BEC state & Sampling of disorder \\
Quantum measurement $\langle\hat{O}(t)\rangle$ & Disorder average $\overline{\langle\hat{o}(t)\rangle}$ \\
\hline \hline
\end{tabular}

where

$$
\left|\left\{\mathbf{r}_{i}\right\}\right\rangle=\frac{\hat{b}_{\mathbf{r}_{1}}^{\dagger} \cdots \hat{b}_{\mathbf{r}_{N}}^{\dagger}}{\sqrt{N !}}|0\rangle
$$

defines the state where the $N$ bosons have well-defined positions $\mathbf{r}_{i}$. The combined state of the system reads

$$
|\Psi\rangle=|\psi\rangle \otimes|\mathrm{BEC}\rangle=\int_{\left\{\mathbf{r}_{i}\right\}}|\psi\rangle \otimes\left|\left\{\mathbf{r}_{i}\right\}\right\rangle .
$$

For infinitely massive bosons $m_{\mathrm{B}} / m_{\mathrm{I}}=\infty$, the time evolution of each state $|\psi\rangle \otimes\left|\left\{\mathbf{r}_{i}\right\}\right\rangle$ contributing to the superposition (4) can be determined exactly. The boson kinetic energy drops out and the total Hamiltonian commutes with the bosonic position operators. Hence, the bosons remain in the state $\left|\left\{\mathbf{r}_{i}\right\}\right\rangle$. Physically, the heavy bosons' positions are not affected by the interaction with the impurity. On the other hand, the impurity views the $N$ localized bosons as scatterers at positions $\mathbf{r}_{i}$ and evolves through the Edwards Hamiltonian (1) such that the system evolves into

$$
|\Psi(t)\rangle=\int_{\left\{\mathbf{r}_{i}\right\}}\left[e^{-i \hat{H}_{\mathrm{Ed}}^{\left\{\mathbf{r}_{i}\right\}}}|\psi\rangle\right] \otimes\left|\left\{\mathbf{r}_{i}\right\}\right\rangle ;
$$

i.e., the system evolves as a superposition over all possible disorder realizations.

Hence, the expectation value of any observable of the impurity $\hat{O}=\hat{o} \otimes 1$ with respect to the state $|\Psi(t)\rangle$,

$$
\langle\hat{O}(t)\rangle=\int_{\left\{\mathbf{r}_{i}\right\}}\left\langle\psi\left|e^{i \hat{H}_{\mathrm{Ed}}^{\left\langle\mathbf{r}_{i}\right\}}} \hat{o} e^{-i \hat{H}_{\mathrm{Ed}}^{\left\{\mathbf{r}_{i}\right\}} t}\right| \psi\right\rangle=\overline{\langle\hat{o}(t)\rangle},
$$

realizes the disorder average $\overline{\langle\hat{o}(t)\rangle}$ [Eq. (2)]. All states in the superposition contribute equally to the measurement of $\hat{O}$, thus carrying out an average over all possible $\left\{\mathbf{r}_{i}\right\}$ configurations. In other words, the fact that the BEC is a quantum superposition with equal weight of orthogonal states $\left|\left\{\mathbf{r}_{i}\right\}\right\rangle$ enables disorder averaging in an analogous way using quantum systems such as ultracold atoms. By contrast with a proposal in Ref. [49] where disorder is simulated using pinned atoms loaded in an optical lattice, in the present work randomness is simulated using the quantum superposition in which the bosons are prepared, similar to a suggestion made for spin systems in Ref. [50].

The correspondence between the two models is summarized in Table I. We emphasize that $\langle\hat{O}(t)\rangle$ and $\overline{\langle\hat{o}(t)\rangle}$ have very different meanings. While $\langle\hat{O}(t)\rangle$ represents a manybody measurement of the impurity evolving through the interaction with a bath of heavy bosons, $\overline{\langle\hat{o}(t)\rangle}$ corresponds to the measurement of the corresponding observable in the 
single-particle Edwards model averaged over many classical realizations of disorder.

\section{GENERALIZATIONS}

Most assumptions made for simplicity in the proof can be relaxed, as long as the crucial ingredient that the $\left|\left\{\mathbf{r}_{i}\right\}\right\rangle$ are eigenstates of the Hamiltonian remains valid. In particular, the proof applies to any dimension. Moreover, it is possible to include arbitrary confining potentials or interactions between the bosons and even prepare the boson bath in a mixed state. In all these cases, the corresponding distribution of disorder would not be uniform but set by the boson state.

Crucially, the mapping holds also for multiple particles. That way, it is possible to simulate transport properties of realistic metals or to consider interactions between the impurities in order to investigate the interplay of many-body and disorder effects. The latter scenario was recently considered in a study of many-body localization, where the thermalization properties of mass-imbalanced mixtures was investigated [51].

The mapping holds when the bosons have a mass exceedingly large compared to the impurity. When this is not the case, the feedback of the interaction with the impurity as well as eventual interactions among bosons will induce dynamics of the bath, defining a characteristic timescale $\tau_{\phi}$ diverging with $m_{\mathrm{B}} / m_{\mathrm{I}}$. In the disorder model, this would correspond to dynamical disorder and cause dephasing. While this may hinder the observation of phenomena associated with disorder, one could conversely take advantage of the presence of $\tau_{\phi}$ to investigate the physics of dephasing in a controlled manner. Furthermore, the paradigm of using a controlled bath as an "auxiliary disorder" can be reversed. Should one be interested in the bosons' properties, the dynamics of the impurity can give information about the time correlations of the bosons, akin to diffusion wave spectroscopy [46,52].

\section{BENCHMARKING VARIATIONAL SOLUTIONS TO BOSE POLARONS}

So far, we have discussed how the exact mapping can provide alternative approaches to disorder theory by enabling the realization of disorder in a controlled manner. However, one can also turn the correspondence around and use disorder theory to gain insight into polaron formation by providing an exactly solvable limit. As an example, we use the mapping to test variational solutions of the Bose polaron problem. While widely used, the quality of such approximations is hard to gauge, given their nonperturbative nature as there is no small control parameter.

Specifically, we consider the one-dimensional Bose polaron described by the Hamiltonian (3) where a single light impurity of mass $m_{\mathrm{I}}$ interacts with a bath of heavy bosons of mass $m_{\mathrm{B}} \gg m_{\mathrm{I}}$ through a contact interaction $v(\mathbf{r})=g \delta(\mathbf{r})$, chosen here to be repulsive $(g>0)$ so that there are no bosonimpurity bound states. We turn our attention to the spread of a particle prepared in a Gaussian wave packet $\psi(\mathbf{r}, t=0) \propto$ $\exp \left(-\mathbf{r}^{2} / 2 \sigma^{2}\right)$. As discussed in Appendix A, the corresponding disorder problem can be solved exactly [48] and can thus serve as a means to assess variational approximations in the limit $m_{\mathrm{B}} / m_{\mathrm{I}}=\infty$.

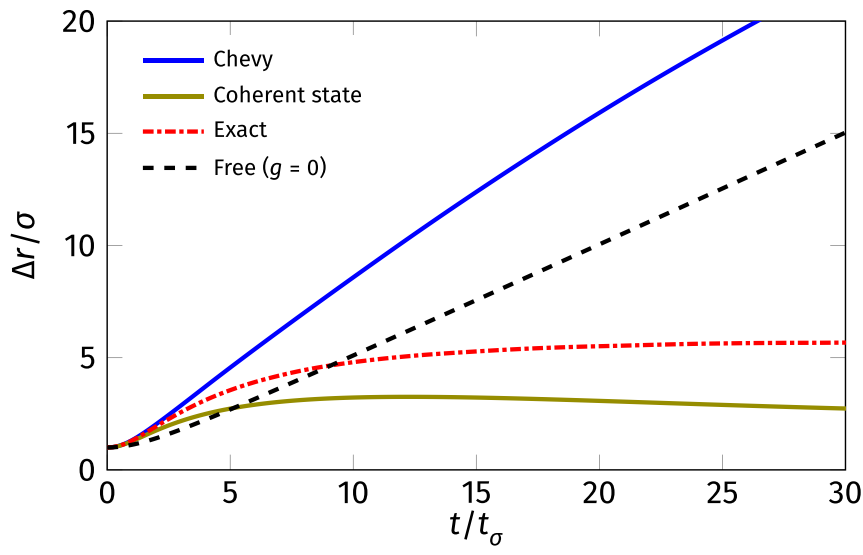

FIG. 2. Time evolution of the width $\Delta r$ of the impurity wave packet. We compare the exact solution (red dash-dotted line) to variational results based on a coherent state (yellow lower solid line) or Chevy Ansatz (blue upper solid line). The initial width of the wave packet $\sigma$ sets a characteristic timescale $t_{\sigma}=2 m_{\mathrm{I}} \sigma^{2} / \hbar$ and the interaction and boson density $n$ are $g / \Omega=1.5 \hbar t_{\sigma}^{-1}$ and $\sigma n=0.4$. The free spread obtained for $g=0$ is shown for reference (black dashed line).

Since all states are localized in the presence of disorder in one dimension, at any finite interaction $g>0$ the wave packet is localized at $t \rightarrow \infty$, i.e., $\psi(\mathbf{r}, t) \rightarrow \exp (-|\mathbf{r}| / 2 \xi)$ at large $|\mathbf{r}|$, with $\xi$ a localization length. This contrasts with the free case $(g=0)$ where the wave packet spreads indefinitely. A simple observable that distinguishes the two regimes is the width of the wave packet $\Delta r=\sqrt{\left\langle(\mathbf{r}-\langle\mathbf{r}\rangle)^{2}\right\rangle}$. For a localized wave packet, $\Delta r$ remains finite at all times, while in the free case $\Delta r$ grows linearly with time.

We first compare the exact solution of the disorder model to the Chevy Ansatz, which includes for the time-dependent wave function $\left|\Psi^{\mathbf{p}}(t)\right\rangle$ at most one bosonic excitation [53,54], i.e.,

$$
\begin{aligned}
\left|\Psi^{\mathbf{p}}(t)\right\rangle= & \alpha_{0}(t)|\mathbf{p}\rangle \otimes|\mathrm{BEC}\rangle \\
& +\sum_{\mathbf{q} \neq 0} \alpha_{\mathbf{q}}(t)|\mathbf{p}+\mathbf{q}\rangle \otimes \hat{b}_{-\mathbf{q}}^{\dagger} \hat{b}_{0}|\mathrm{BEC}\rangle,
\end{aligned}
$$

where $\alpha_{\mathbf{q}}(t)$ are time-dependent variational parameters (for details see Appendix B).

The time-resolved spreading of the wave packet is compared to the exact solution in Fig. 2. At short times, we observe good agreement with the exact solution. This is to be expected as, in variational methods, the deviation from the exact solution arises from the iterated projection of the Schrödinger equation and the associated error did not built up at early times. This is also reflected in the evolution of the spatially resolved wave packet shown in Fig. 3, where the whole density profile is well described by the Chevy Ansatz at short times. Since the Chevy Ansatz specifically relies on including only a small number of excitations, it is most accurate at short times at which the polaron cloud is still developing. However, the Chevy Ansatz breaks down at larger times and, rather than the localization clearly observed in the exact solution, it predicts the indefinite spread of the wave packet. 

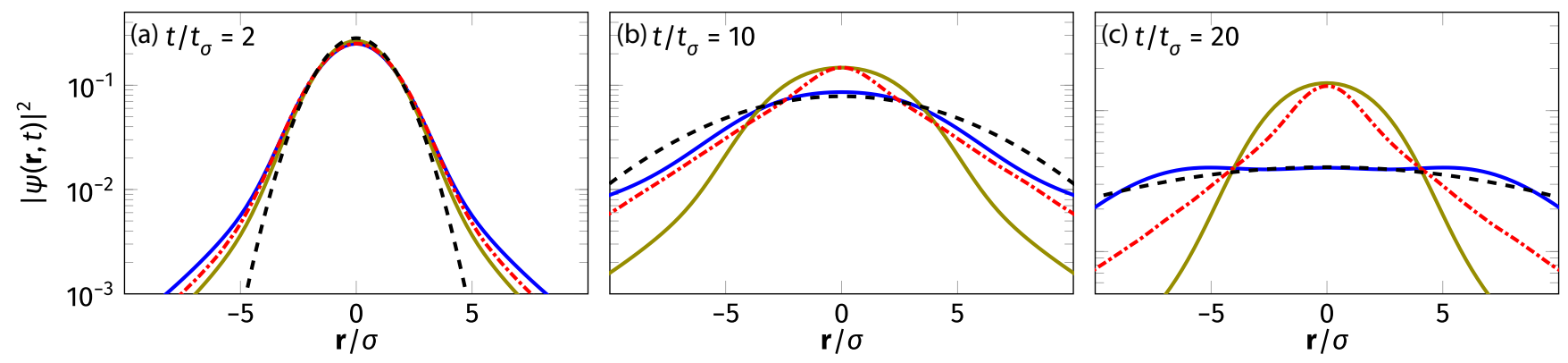

FIG. 3. Density profile $|\psi(\mathbf{r}, t)|^{2}$ as a function of $\mathbf{r}$ of the impurity interacting with a BEC for increasing times $t$ : (a) $t / t_{\sigma}=2$, (b) $t / t_{\sigma}=10$, and (c) $t / t_{\sigma}=20$. We compare the exact solution (red dash-dotted line) to the variational result based on a coherent state (yellow initially lower solid line) and Chevy Ansatz (blue initially upper solid line). We use $\sigma n=0.4$ and $g / \Omega=1.5 \hbar t_{\sigma}^{-1}$. The free spread ( $g=0$ ) is shown for reference (black dashed line).

This prompts the search for more involved variational solutions. We consider an Ansatz based on the Lee-Low-Pines transformation [55] to transform into the comoving frame of the impurity. In the new frame the impurity momentum $\mathbf{p}$ represents the conserved total momentum of the complete system. Impurity operators are hence eliminated and the bosons evolve according to the $\mathbf{p}$-dependent Hamiltonian

$$
\hat{H}_{\mathbf{p}}^{\mathrm{BEC}}=\frac{\left(\mathbf{p}-\sum_{\mathbf{k}} \mathbf{k} \hat{b}_{\mathbf{k}}^{\dagger} \hat{b}_{\mathbf{k}}\right)^{2}}{2 m_{\mathrm{I}}}+\sum_{\mathbf{k}} \omega_{\mathbf{k}} \hat{b}_{\mathbf{k}}^{\dagger} \hat{b}_{\mathbf{k}}+\frac{g}{\Omega} \sum_{\mathbf{k} \mathbf{k}^{\prime}} \hat{b}_{\mathbf{k}}^{\dagger} \hat{b}_{\mathbf{k}^{\prime}}
$$

which now contains a transformation-induced interaction between bosons. Here $\Omega$ is the system volume.

The Hamiltonian (10) has been studied using various $A n$ sätze [56-63]. We approximate the boson wave function $\left|\Phi^{\mathbf{p}}(t)\right\rangle$ by a product of coherent states,

$$
\left|\Phi^{\mathbf{p}}(t)\right\rangle=e^{-i \phi^{\mathbf{p}}(t)} \exp \left(\sum_{\mathbf{k}} \beta_{\mathbf{k}}^{\mathbf{p}}(t) \hat{b}_{\mathbf{k}}^{\dagger}-\text { H.c. }\right)|0\rangle
$$

with variational parameters $\phi^{\mathbf{p}}(t)$ and $\beta_{\mathbf{k}}^{\mathbf{p}}(t)$. By considering this wave function, we neglect the possibility of correlations induced between the infinitely massive bosons.

The results for the wave packet are shown in Figs. 3 and 2. Again, the exact solution is well reproduced at short times, unsurprisingly, since for a state close to the BEC, Eq. (11) reduces to Eq. (9). However, the coherent Ansatz directly addresses one of the shortcomings of the Chevy Ansatz by allowing the description of a large number of bosons excited to small momenta and thus captures localization, although $\xi$ is underestimated.

These examples demonstrate how the exact mapping can help benchmark variational solutions by providing an exact reference solution of the polaron problem. Indeed, both the Chevy and coherent Ansätze can be improved systematically, respectively, by allowing more bosonic excitations [10-13] or by considering Gaussian states [64] with the mapping allowing one to quantify the improvement. This equally applies to other schemes such as non-Gaussian states or quantum Monte Carlo [65-70].

\section{APPLICATION TO THE ANDERSON TRANSITION}

A long-standing question in disorder physics is that of the Anderson transition in three dimensions (3D). Unlike in one dimension, where all states are localized, in 3D a mobility edge separates low-energy localized and high-energy extended states [28]. The observation of the mobility edge using the expansion of atomic matter waves through disordered speckle potentials has sparked recent theoretical [71,72] and experimental interest $[41,73,74]$. Discrepancies between theory and experiments remain, however, as to the position of the mobility edge $[72,75]$. On the theory side, the problem is difficult because it requires going beyond the perturbative weak-disorder regime and treating fully the speckle potential, while for experiments it is difficult to prepare a sufficiently narrow energy distribution of the atomic cloud.

The polaron-disorder correspondence can help us obtain further insight. A specific candidate is ${ }^{6} \mathrm{Li}$ impurities embedded in ${ }^{133} \mathrm{Cs}$ with $m_{\mathrm{B}} / m_{\mathrm{I}}=22.1$ [76,77]. In particular, the $\mathrm{Li}-\mathrm{Cs}$ interaction can be tuned by a Feshbach resonance at $889 \mathrm{G}$ at which the Cs-Cs interaction is small $\left(a_{\mathrm{CsCs}} \simeq 190 a_{0}\right)$. The cloud of impurities expands through the bosonic medium as through a disordered Edwards potential. Theorywise, one does not have to deal with the speckle potential and can use the arsenal developed to tackle strongly interacting problems to study the dynamics of the mixture. Nonetheless, the question of the determination of the mobility edge remains an ambitious one. In order to answer it, two key aspects have to be addressed. The first is given by the aforementioned decoherence induced by the finite boson mass. The second constraint is given by the lifetime of the system set, e.g., by three-body loss. This may guide the choice of the system and, for instance, molecular mixtures that feature suppressed three-body loss might prove better candidates despite having lower mass ratios than Li-Cs [78].

\section{CONCLUSION AND OUTLOOK}

We have shown that a generic model of disorder, the Edwards model, is mapped to the problem of a light impurity coupled to a BEC, showing a deep connection between two seemingly very different problems. Our exact mapping has several implications. Experimentally, it offers an alternative setup to investigate the Anderson transition. On the theory 
side, it can help to develop reliable approximation schemes to describe the Bose polaron problem while conversely offering an efficient way to bypass the challenging issue of disorder averaging. However, the mapping applies to a much larger class of models. For instance, in the case of a mixture with both interactions and disorder, the mapping can be used to gauge away interactions, reducing it to a single-particle problem in the presence of two sources of disorder. Another possibility is the realization of more complicated models of disorder, e.g., with long-range correlations, by tuning the boson Hamiltonian.

A promising avenue of research could originate from the field-theoretic treatment of the Bose polaron problem. For instance, the Chevy Ansatz is equivalent to a non-self-consistent $T$-matrix approach $[53,54]$. It would be interesting to see how such methods compare to the diagrammatic treatment of disorder and whether they can be used to shed light on either side of the mapping. Further intriguing applications would be the study of the interplay of disorder and interactions, especially in lower dimensions where many-body methods such as the use of variational matrix product states could help us understand thermalization and the spread of entanglement [79-82]. A recent proposal of a different mapping between disordered systems and interacting semimetals brings together disorder and many-body physics in a solid-state setting [83]. While our work focuses on cold-atom applications, the mapping presented in this work can be extended to other condensed-matter systems. Indeed, the crucial ingredient behind the mapping is the absence of boson kinetic energy. Hence, solid-state realizations using as a bath a system with flat bands, such as encountered within the quantum Hall effect, are also conceivable.

\section{ACKNOWLEDGMENTS}

The authors thank Cheng Chin, Arthur Christianen, Eugene Demler, Falko Pientka, Lode Pollet, Tao Shi, Binh Tran, Matteo Zacanti, and Wilhelm Zwerger for fruitful discussions. They are grateful to Jean-Marc Luck for his insight regarding the RKPM and pointing out Ref. [48]. We acknowledge support from the Deutsche Forschungsgemeinschaft (German Research Foundation) under Germany's Excellence Strategy EXC-2111-390814868.

\section{APPENDIX A: EXACT SOLUTION OF THE RKPM}

The random Kronig-Penney model is defined by the onedimensional Hamiltonian

$$
\hat{H}_{\mathrm{RKPM}}=-\frac{\partial_{x}^{2}}{2 m}+g \sum_{i=1}^{N} \delta\left(x-x_{i}\right),
$$

describing a single particle in a box of size $L$ with mass $m$ and position $x \in[0, L]$ interacting with fixed random scatterers with a contact potential of strength $g$, with $\hbar$ set equal to 1. We consider a given realization of disorder defined by the positions of the $N$ scatterers $x_{i}$, which we choose to be enumerated as $x_{i}<x_{i+1}$ and note for convenience that $x_{0}=0$, $x_{N+1}=L$.
We seek to solve the Schrödinger equation

$$
E \psi(x)=-\frac{1}{2 m} \psi^{\prime \prime}(x)+g \sum_{i=1}^{N} \delta\left(x-x_{i}\right) \psi(x) .
$$

The general solution is found using a standard transfer matrix method (see, e.g., Ref. [48]). We consider only the case of a repulsive scattering potential $g>0$, for which $E$ is positive, as can been seen by multiplying Eq. (A2) by $\psi(x)^{*}$ and integrating over the box. On any subinterval $] x_{i}, x_{i+1}[$ the eigenfunctions $\psi(x)$ take the form

$$
\psi(x)=A_{i} \sin \left(k x+\phi_{i}\right),
$$

with $A_{i}$ and $\phi_{i}$ an amplitude and a phase, respectively, and $k \geqslant 0$ defined by $E=k^{2} / 2 m$. While $\psi$ is continuous, there is a jump in $\psi^{\prime}$ at each scatterer $x_{i}$,

$$
\psi^{\prime}\left(x_{i}^{+}\right)-\psi^{\prime}\left(x_{i}^{-}\right)=2 m g \psi\left(x_{i}\right),
$$

as can been seen by integrating Eq. (A2) over $\left[x_{i}^{-}, x_{i}^{+}\right]$.

Constructing the vector $\Psi(x)=\left(\psi^{\prime}(x), k \psi(x)\right)^{\top}$ indexed by the coordinate $x$, the conditions (A3) and (A4) can be rewritten as

$$
\Psi\left(x_{i+1}^{-}\right)=R\left[k\left(x_{i+1}-x_{i}\right)\right] \Psi\left(x_{i}^{+}\right), \quad \Psi\left(x_{i}^{+}\right)=T \Psi\left(x_{i}^{-}\right),
$$

where

$$
R(\theta)=\left(\begin{array}{cc}
\cos (\theta) & -\sin (\theta) \\
\sin (\theta) & \cos (\theta)
\end{array}\right), \quad T=\left(\begin{array}{cc}
1 & \frac{2 m g}{k} \\
0 & 1
\end{array}\right) .
$$

Hence, $\Psi(L)=M \Psi(0)$, with

$$
M=R\left[k\left(L-x_{N}\right)\right] T R\left[k\left(x_{N}-x_{N-1}\right)\right] \cdots T R\left[k x_{1}\right] .
$$

The spectrum is fixed by the boundary conditions; for instance, periodic boundary conditions impose $M=1$ or equivalently $\operatorname{Tr} M=2$, as $\operatorname{det} M=1$.

Once the spectrum is determined, one finds for each eigenvalue the amplitudes and phases $A_{i}$ and $\phi_{i}$ defining the eigenstate. Using the continuity of $\psi(x)$ together with Eq. (A4), one relates $A_{i+1}$ and $\phi_{i+1}$ to $A_{i}$ and $\phi_{i}$. Thus, by recursion, $A_{N}$ and $\phi_{N}$ can be expressed as functions of $\phi_{0}$ and $A_{0}$. Finally, a suitable value of $\phi_{0}$ is determined numerically such that the boundary condition is fulfilled, while the amplitudes are fixed by the normalization. As an illustration, we show the first eigenstates thus obtained for a given realization of disorder in Fig. 4.

For attractive interactions $(g<0)$, not considered in this article, this method remains valid. However, in this case one also needs to consider eigenstates of negative energy $E=$ $-\kappa^{2} / 2 m$. On each subinterval, the corresponding wave functions then take the form $\psi(x)=A_{i} e^{-\kappa x}+B_{i} e^{\kappa x}$, physically representing states that are exponentially localized about the scatterers. The spectrum and wave functions can again be determined by the transfer matrix method.

\section{APPENDIX B: TIME-DEPENDENT VARIATIONAL METHOD}

In this Appendix we briefly review how to determine the time evolution of a ket $|\psi\rangle$ under a Hamiltonian $\hat{H}$ using 

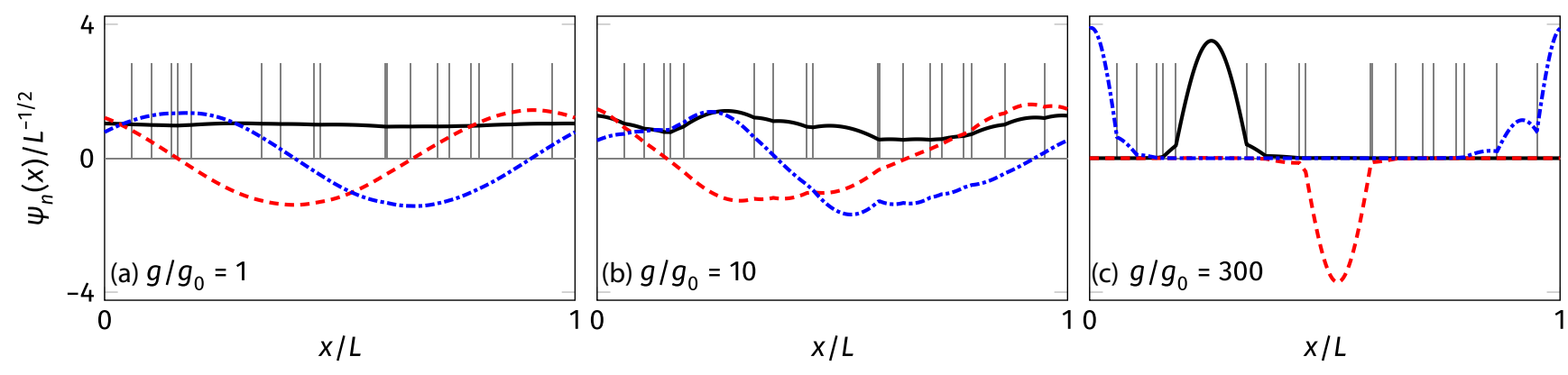

FIG. 4. First three eigenstates $\psi_{n=1,2,3}(x)$ of $\hat{H}_{\mathrm{RPKM}}$ for a given realization of disorder for three different interaction strengths $g$, (a) $g / g_{0}=1$, (b) $g / g_{0}=10$, and (c) $g / g_{0}=300$, expressed in units of $g_{0}=(2 m L)^{-1}$. The ground-state wave function and the first two excited states are shown as black solid, red dashed, and blue dash-dotted lines, respectively. We use periodic boundary conditions in a box of size $L$. The positions of the scatterers $x_{i}$ are represented by gray vertical lines. For weak disorder (a) the wave functions closely resemble the solutions of a free particle in a box, while at larger interactions (b) and (c) the eigenstates become localized over distances smaller than the box size.

the variational method (see Refs. [64,84-87] for a more indepth discussion). We first provide a general discussion before applying the formalism to the variational states (9) and (11) introduced in the main text.

We seek the best approximation of the Schrödinger equation $\left(i \partial_{t}-\hat{H}\right)|\psi\rangle=0$, with $|\psi\rangle$ being restrained to a variational manifold $\mathcal{M}$. Let us assume that $\mathcal{M}=\left\{\left|\psi\left(z_{i}\right)\right\rangle, z_{i} \in\right.$ $\mathbb{C}\}$ is parametrized by complex numbers $z_{i}$, with $\left|\psi\left(z_{i}\right)\right\rangle$ a holomorphic function of the $z_{i}$. Under that assumption, the following three approaches yield the same equations of motions for the variational parameters $z_{i}$. The first is to minimize at all times the norm of the ket $\left(i \partial_{t}-\hat{H}\right)|\psi\rangle$ with respect to the $\dot{z}_{i}$. The second approach is to extremize the Lagrangian

$$
L=\frac{i}{2}\left[\langle\psi|\left(\partial_{t}|\psi\rangle\right)-\left(\partial_{t}\langle\psi|\right)|\psi\rangle\right]-\langle\psi|\hat{H}| \psi\rangle
$$

with respect to the variational parameters $\left(z_{i}, \dot{z}_{i}\right)$. The third approach is to project at every time step the Schrödinger equation onto the tangent space to $\mathcal{M}$. We use in practice the last method, which we present below.

\section{Gram matrix formulation}

The tangent space to $\mathcal{M}$ at $|\psi\rangle$ is spanned by the vectors

$$
\left|\partial_{i} \psi\right\rangle=\frac{\partial}{\partial z_{i}}|\psi(z)\rangle
$$

Hence the projected Schrödinger equation is satisfied if and only if for all $i$,

$$
\left\langle\partial_{i} \psi\left|\left(i \partial_{t}-\hat{H}\right)\right| \psi\right\rangle=0 .
$$

We now define the energy of the state $E$ and the Gram matrix $G$ using the overlaps of the vectors $\left|\partial_{i} \psi\right\rangle$,

$$
\begin{gathered}
E=\langle\psi|\hat{H}| \psi\rangle, \\
E_{j}=\frac{\partial}{\partial z_{j}^{*}} E=\left\langle\partial_{j} \psi|\hat{H}| \psi\right\rangle, \\
G_{i j}=\left\langle\partial_{i} \psi \mid \partial_{j} \psi\right\rangle .
\end{gathered}
$$

Equation (B3) can then be rewritten as

$$
\sum_{j} i G_{i j} \dot{z}_{j}=E_{i}
$$

In the specific case where $G$ is invertible, the equivalent form is

$$
i \dot{z}_{i}=\sum_{j}\left[G^{-1}\right]_{i j} E_{j}
$$

Otherwise there is some indeterminacy in the equations of motion, i.e., it is possible to find two different solutions $\dot{z}_{i}$ and $\dot{z}_{i}^{\prime}$ fulfilling Eq. (B7) provided $G_{i j}\left(\dot{z}_{j}-\dot{z}_{j}^{\prime}\right)=0$.

\section{Application to coherent states}

While determining the equations of motion using the Gram formalism for the Chevy Ansatz (9) is straightforward, the case of the coherent state Ansatz is slightly more involved, and we present here the detailed derivation. Indeed, when applying the Gram formalism, one encounters two issues for the coherent Ansatz (11). First, it is not holomorphic (as both $\beta_{\mathbf{k}}$ and $\beta_{\mathbf{k}}^{*}$ appear), and second, there is no straightforward equation of motion for the phase, which represents a gauge degree of freedom.

We tackle both issues at once by rather considering the variational state

$$
|\beta\rangle=\mathcal{N} \exp \left(\sum_{\mathbf{k}} \beta_{\mathbf{k}} \hat{b}_{\mathbf{k}}^{\dagger}\right)|0\rangle,
$$

parametrized by the complex numbers $\mathcal{N}$ and $\beta_{\mathbf{k}}$, with $\mathcal{N}$ introduced for normalization. The Ansätze (11) and (B8) are completely equivalent provided $\mathcal{N}=\exp (-i \phi-$ $\left.\sum_{\mathbf{k}}\left|\beta_{\mathbf{k}}\right|^{2} / 2\right)$.

For clarity, we drop in this section the $t$ and $\mathbf{p}$ dependences. We rewrite Eq. (B8) by defining the vectors $(\boldsymbol{\beta})_{\mathbf{k}}=\beta_{\mathbf{k}}$ and $(\hat{\mathbf{b}})_{\mathbf{k}}=\hat{b}_{\mathbf{k}}$ such that $|\beta\rangle=\mathcal{N} e^{\hat{\mathbf{b}}^{\dagger} \cdot \boldsymbol{\beta}}|0\rangle$. We also note that $S=$ $(\boldsymbol{\beta})^{\dagger} \cdot \boldsymbol{\beta}=\sum_{\mathbf{k}}\left|\beta_{\mathbf{k}}\right|^{2}$.

We use the Gram matrix formulation where, from the gradients of $|\beta\rangle$,

$$
\begin{aligned}
& \left|\partial_{\mathcal{N}} \beta\right\rangle=\frac{\partial}{\partial \mathcal{N}}|\beta\rangle=\frac{1}{\mathcal{N}}|\beta\rangle, \\
& \left|\partial_{\mathbf{k}} \beta\right\rangle=\frac{\partial}{\partial \beta_{\mathbf{k}}}|\beta\rangle=\hat{b}_{\mathbf{k}}^{\dagger}|\beta\rangle,
\end{aligned}
$$


we deduce the Gram matrix [Eq. (B5)]

$$
G=\left(\begin{array}{cc}
1 & \mathcal{N} \boldsymbol{\beta}^{\dagger} \\
\mathcal{N}^{*} \boldsymbol{\beta} & |\mathcal{N}|^{2}\left(\delta+\boldsymbol{\beta} \cdot \boldsymbol{\beta}^{\dagger}\right)
\end{array}\right) e^{S}
$$

In the above expression, the first row and column stand for the $\mathcal{N}$ direction, while the rest of the matrix corresponds to the momentum modes labeled by $\mathbf{k}$. In the $\mathbf{k - k}$ sector, $\delta$ is understood as the identity matrix and $\boldsymbol{\beta} \cdot \boldsymbol{\beta}^{\dagger}$ as the matrix with elements $\left(\boldsymbol{\beta} \cdot \boldsymbol{\beta}^{\dagger}\right)_{\mathbf{k}, \mathbf{k}^{\prime}}=\beta_{\mathbf{k}} \beta_{\mathbf{k}^{\prime}}^{*}$. Here $G$ is invertible with the inverse

$$
G^{-1}=\left(\begin{array}{cc}
1+S & -\frac{1}{\mathcal{N}^{*}} \boldsymbol{\beta}^{\dagger} \\
-\frac{1}{\mathcal{N}} \boldsymbol{\beta} & \frac{1}{|\mathcal{N}|^{2}} \delta
\end{array}\right) e^{-S} .
$$

The energy [Eq. (B4)] is given by

$$
E=|\mathcal{N}|^{2} e^{S} E_{0},
$$

where

$$
E_{0}=\sum_{\mathbf{k}}\left(\epsilon_{\mathbf{k}}+\omega_{\mathbf{k}}\right)\left|\beta_{\mathbf{k}}\right|^{2}+\frac{\left(\mathbf{p}-\mathbf{P}_{\mathrm{B}}[\boldsymbol{\beta}]\right)^{2}}{2 m}+\frac{g}{\Omega}\left|\sum_{\mathbf{q}} \beta_{\mathbf{q}}\right|^{2}
$$

and $\mathbf{P}_{\mathrm{B}}[\boldsymbol{\beta}]=\sum_{\mathbf{k}} \mathbf{k}\left|\beta_{\mathbf{k}}\right|^{2}$ is the total momentum of the bosons. The gradients of $E$ read

$$
\begin{gathered}
E_{\mathcal{N}}=\frac{\partial}{\partial \mathcal{N}^{*}} E=\mathcal{N} e^{S} E_{0}, \\
E_{\mathbf{k}}=\frac{\partial}{\partial \beta_{\mathbf{k}}^{*}} E=|\mathcal{N}|^{2} e^{S}\left\{\beta_{\mathbf{k}} E_{0}\right. \\
\left.+\left[\left(\epsilon_{\mathbf{k}}+\omega_{\mathbf{k}}-\mathbf{k} \cdot \frac{\mathbf{p}-\mathbf{P}_{\mathrm{B}}[\boldsymbol{\beta}]}{m}\right) \beta_{\mathbf{k}}+\frac{g}{\Omega} \sum_{\mathbf{q}} \beta_{\mathbf{q}}\right]\right\}
\end{gathered}
$$

Applying Eq. (B7), we obtain

$$
\begin{gathered}
i \dot{\beta}_{\mathbf{k}}=\left(\epsilon_{\mathbf{k}}+\omega_{\mathbf{k}}-\mathbf{k} \cdot \frac{\mathbf{p}-\mathbf{P}_{\mathrm{B}}[\boldsymbol{\beta}]}{m}\right) \beta_{\mathbf{k}}+\frac{g}{\Omega} \sum_{\mathbf{q}} \beta_{\mathbf{q}}, \\
i \dot{\mathcal{N}}=\mathcal{N} \frac{\mathbf{p}^{2}-\mathbf{P}_{\mathrm{B}}[\boldsymbol{\beta}]^{2}}{2 m} .
\end{gathered}
$$

From these equations of motion we deduce that $|\mathcal{N}|$ and $S$ are constant in time, as expected since the Hamiltonian conserves the number of particles and $S=\langle\beta|\hat{N}| \beta\rangle /\langle\beta \mid \beta\rangle=N$. Hence the norm of $|\beta\rangle,\langle\beta \mid \beta\rangle=|\mathcal{N}|^{2} e^{S}$, is conserved. We can rewrite $\mathcal{N}=\exp (-S / 2) \exp (-i \phi)$ as

$$
\dot{\phi}=\frac{\mathbf{p}^{2}-\mathbf{P}_{\mathrm{B}}[\boldsymbol{\beta}]^{2}}{2 m_{\mathrm{I}}} .
$$

Using this new notation, Eq. (B8) is exactly equivalent to (11). The equations of motion thus obtained are the same as those present in the literature (see, e.g., Ref. [58]).
[1] L. Landau and S. Pekar, Effective mass of a polaron, J. Exp. Theor. Phys. 18, 419 (1948).

[2] C. B. Duke and G. D. Mahan, Phonon-broadened impurity spectra. I. Density of states, Phys. Rev. 139, A1965 (1965).

[3] A. S. Alexandrov and N. F. Mott, Polarons and Bipolarons (World Scientific, Singapore, 1995).

[4] G. Baym and C. Pethick, Landau Fermi-Liquid Theory: Concepts and Applications (Wiley-VCH, New York, 1991).

[5] M. E. Gershenson, V. Podzorov, and A. F. Morpurgo, Colloquium: Electronic transport in single-crystal organic transistors, Rev. Mod. Phys. 78, 973 (2006).

[6] E. Dagotto, Correlated electrons in high-temperature superconductors, Rev. Mod. Phys. 66, 763 (1994).

[7] J. T. Devreese and A. S. Alexandrov, Fröhlich polaron and bipolaron: Recent developments, Rep. Prog. Phys. 72, 066501 (2009).

[8] A. G. Volosniev, H.-W. Hammer, and N. T. Zinner, Real-time dynamics of an impurity in an ideal Bose gas in a trap, Phys. Rev. A 92, 023623 (2015).

[9] M. A. García-March, A. S. Dehkharghani, and N. T. Zinner, Entanglement of an impurity in a few-body one-dimensional ideal Bose system, J. Phys. B 49, 075303 (2016).

[10] J. Levinsen, M. M. Parish, and G. M. Bruun, Impurity in a Bose-Einstein Condensate and the Efimov Effect, Phys. Rev. Lett. 115, 125302 (2015).

[11] S. M. Yoshida, S. Endo, J. Levinsen, and M. M. Parish, Universality of an Impurity in a Bose-Einstein Condensate, Phys. Rev. X 8, 011024 (2018).
[12] Z.-Y. Shi, S. M. Yoshida, M. M. Parish, and J. Levinsen, Impurity-Induced Multibody Resonances in a Bose Gas, Phys Rev. Lett. 121, 243401 (2018).

[13] J. Levinsen, L. A. Peña Ardila, S. M. Yoshida, and M. M. Parish, Quantum Behavior of a Heavy Impurity Strongly Coupled to a Bose Gas, Phys. Rev. Lett. 127, 033401 (2021).

[14] A. S. Dehkharghani, A. G. Volosniev, and N. T. Zinner, Coalescence of Two Impurities in a Trapped One-dimensional Bose Gas, Phys. Rev. Lett. 121, 080405 (2018).

[15] J. Jager, R. Barnett, M. Will, and M. Fleischhauer, Strongcoupling Bose polarons in one dimension: Condensate deformation and modified Bogoliubov phonons, Phys. Rev. Research 2, 033142 (2020).

[16] D. Dzsotjan, R. Schmidt, and M. Fleischhauer, Dynamical Variational Approach to Bose Polarons at Finite Temperatures, Phys. Rev. Lett. 124, 223401 (2020).

[17] N.-E. Guenther, R. Schmidt, G. M. Bruun, V. Gurarie, and P. Massignan, Mobile impurity in a Bose-Einstein condensate and the orthogonality catastrophe, Phys. Rev. A 103, 013317 (2021).

[18] P. Massignan, N. Yegovtsev, and V. Gurarie, Universal Aspects of a Strongly Interacting Impurity in a Dilute Bose Condensate, Phys. Rev. Lett. 126, 123403 (2021).

[19] F. Isaule, I. Morera, P. Massignan, and B. Juliá-Díaz, Renormalization-group study of Bose polarons, Phys. Rev. A 104, 023317 (2021).

[20] R. Schmidt and T. Enss, Self-stabilized Bose polarons, arXiv:2102.13616. 
[21] C. Franchini, M. Reticcioli, M. Setvin, and U. Diebold, Polarons in materials, Nat. Rev. Mater. 6, 560 (2021).

[22] K. Seetharam, Y. Shchadilova, F. Grusdt, M. B. Zvonarev, and E. Demler, Dynamical Quantum Cherenkov Transition of Fast Impurities in Quantum Liquids, Phys. Rev. Lett. 127, 185302 (2021).

[23] M.-G. Hu, M. J. Van de Graaff, D. Kedar, J. P. Corson, E. A. Cornell, and D. S. Jin, Bose Polarons in the Strongly Interacting Regime, Phys. Rev. Lett. 117, 055301 (2016).

[24] N. B. Jørgensen, L. Wacker, K. T. Skalmstang, M. M. Parish, J. Levinsen, R. S. Christensen, G. M. Bruun, and J. J. Arlt, Observation of Attractive and Repulsive Polarons in a BoseEinstein Condensate, Phys. Rev. Lett. 117, 055302 (2016).

[25] Z. Z. Yan, Y. Ni, C. Robens, and M. W. Zwierlein, Bose polarons near quantum criticality, Science 368, 190 (2020).

[26] L. A. Peña Ardila, N. B. Jørgensen, T. Pohl, S. Giorgini, G. M. Bruun, and J. J. Arlt, Analyzing a Bose polaron across resonant interactions, Phys. Rev. A 99, 063607 (2019).

[27] M. G. Skou, T. G. Skov, N. B. Jørgensen, K. K. Nielsen, A. Camacho-Guardian, T. Pohl, G. M. Bruun, and J. J. Arlt, Non-equilibrium quantum dynamics and formation of the Bose polaron, Nat. Phys. 17, 731 (2021).

[28] P. W. Anderson, Absence of diffusion in certain random lattices, Phys. Rev. 109, 1492 (1958).

[29] D. Y. Sharvin and Y. V. Sharvin, Magnetic-flux quantization in a cylindrical film of a normal metal, J. Exp. Theor. Phys. Lett. 34, 272 (1981).

[30] B. Pannetier, J. Chaussy, R. Rammal, and P. Gandit, Magnetic Flux Quantization in the Weak-Localization Regime of a Nonsuperconducting Metal, Phys. Rev. Lett. 53, 718 (1984).

[31] Y. Kuga and A. Ishimaru, Retroreflectance from a dense distribution of spherical particles, J. Opt. Soc. Am. A 1, 831 (1984).

[32] P.-E. Wolf and G. Maret, Weak Localization and Coherent Backscattering of Photons in Disordered Media, Phys. Rev. Lett. 55, 2696 (1985).

[33] M. P. Van Albada and Ad. Lagendijk, Observation of Weak Localization of Light in a Random Medium, Phys. Rev. Lett. 55, 2692 (1985).

[34] L. Fleishman and P. W. Anderson, Interactions and the Anderson transition, Phys. Rev. B 21, 2366 (1980).

[35] D. M. Basko, I. L. Aleiner, and B. L. Altshuler, Metal insulator transition in a weakly interacting many-electron system with localized single-particle states, Ann. Phys. (NY) 321, 1126 (2006).

[36] V. Oganesyan and D. A. Huse, Localization of interacting fermions at high temperature, Phys. Rev. B 75, 155111 (2007).

[37] R. Nandkishore and D. A. Huse, Many-body localization and thermalization in quantum statistical mechanics, Annu. Rev. Condens. Matter Phys. 6, 15 (2015).

[38] R. Dalichaouch, J. P. Armstrong, S. Schultz, P. M. Platzman, and S. L. McCall, Microwave localization by two-dimensional random scattering, Nature (London) 354, 53 (1991).

[39] L. Ye, G. Cody, M. Zhou, P. Sheng, and A. N. Norris, Observation of Bending Wave Localization and Quasi Mobility Edge in Two Dimensions, Phys. Rev. Lett. 69, 3080 (1992).

[40] J. Billy, V. Josse, Z. Zuo, A. Bernard, B. Hambrecht, P. Lugan, D. Clément, L. Sanchez-Palencia, P. Bouyer, and A. Aspect, Direct observation of Anderson localization of matter waves in a controlled disorder, Nature (London) 453, 891 (2008).
[41] F. Jendrzejewski, A. Bernard, K. Müller, P. Cheinet, V. Josse, M. Piraud, L. Pezzé, L. Sanchez-Palencia, A. Aspect, and P. Bouyer, Three-dimensional localization of ultracold atoms in an optical disordered potential, Nat. Phys. 8, 398 (2012).

[42] A. Eckardt and E. Anisimovas, High-frequency approximation for periodically driven quantum systems from a Floquet-space perspective, New J. Phys. 17, 093039 (2015).

[43] C. Hainaut, A. Rançon, J.-F. Clément, I. Manai, P. Szriftgiser, D. Delande, J. C. Garreau, and R. Chicireanu, Experimental realization of an ideal Floquet disordered system, New J. Phys. 21, 035008 (2019).

[44] S. Fishman, D. R. Grempel, and R. E. Prange, Chaos, Quantum Recurrences, and Anderson Localization, Phys. Rev. Lett. 49, 509 (1982).

[45] S. F. Edwards, A new method for the evaluation of electric conductivity in metals, Philos. Mag. 3, 1020 (1958).

[46] E. Akkermans and G. Montambaux, Mesoscopic Physics of Electrons and Photons (Cambridge University Press, Cambridge, 2007).

[47] M. Lax and J. C. Phillips, One-dimensional impurity bands, Phys. Rev. 110, 41 (1958).

[48] T. Nieuwenhuizen, Exact electronic spectra and inverse localization lengths in one-dimensional random systems: I. Random alloy, liquid metal and liquid alloy, Physica A 120, 468 (1983).

[49] U. Gavish and Y. Castin, Matter-Wave Localization in Disordered Cold Atom Lattices, Phys. Rev. Lett. 95, 020401 (2005).

[50] B. Paredes, F. Verstraete, and J. I. Cirac, Exploiting Quantum Parallelism to Simulate Quantum Random Many-Body Systems, Phys. Rev. Lett. 95, 140501 (2005).

[51] T. Grover and M. P. A. Fisher, Quantum disentangled liquids, J. Stat. Mech. (2014) 10010.

[52] B. J. Berne and R. Pecora, Dynamic Light Scattering with Applications to Chemistry, Biology and Physics (Wiley, New York, 1976).

[53] S. P. Rath and R. Schmidt, Field-theoretical study of the Bose polaron, Phys. Rev. A 88, 053632 (2013).

[54] W. Li and S. Das Sarma, Variational study of polarons in BoseEinstein condensates, Phys. Rev. A 90, 013618 (2014).

[55] T. D. Lee, F. E. Low, and D. Pines, The motion of slow electrons in a polar crystal, Phys. Rev. 90, 297 (1953).

[56] A. Shashi, F. Grusdt, D. A. Abanin, and E. Demler, Radiofrequency spectroscopy of polarons in ultracold Bose gases, Phys. Rev. A 89, 053617 (2014).

[57] F. Grusdt, A. Shashi, D. Abanin, and E. Demler, Bloch oscillations of bosonic lattice polarons, Phys. Rev. A 90, 063610 (2014).

[58] Y. E. Shchadilova, R. Schmidt, F. Grusdt, and E. Demler, Quantum Dynamics of Ultracold Bose Polarons, Phys. Rev. Lett. 117, 113002 (2016).

[59] B. Kain and H. Y. Ling, Hartree-Fock treatment of Fermi polarons using the Lee-Low-Pine transformation, Phys. Rev. A 96, 033627 (2017).

[60] E. Nakano, H. Yabu, and K. Iida, Bose-Einstein-condensate polaron in harmonic trap potentials in the weak-coupling regime: Lee-Low-Pines-type approach, Phys. Rev. A 95, 023626 (2017).

[61] E. Yakaboylu, B. Midya, A. Deuchert, N. Leopold, and M. Lemeshko, Theory of the rotating polaron: Spectrum and selflocalization, Phys. Rev. B 98, 224506 (2018). 
[62] M. Drescher, M. Salmhofer, and T. Enss, Real-space dynamics of attractive and repulsive polarons in Bose-Einstein condensates, Phys. Rev. A 99, 023601 (2019).

[63] M. Drescher, M. Salmhofer, and T. Enss, Theory of a resonantly interacting impurity in a Bose-Einstein condensate, Phys. Rev. Research 2, 032011(R) (2020).

[64] T. Shi, E. Demler, and J. I. Cirac, Variational study of fermionic and bosonic systems with non-Gaussian states: Theory and applications, Ann. Phys. (NY) 390, 245 (2018).

[65] L. A. Peña Ardila and S. Giorgini, Impurity in a Bose-Einstein condensate: Study of the attractive and repulsive branch using quantum Monte Carlo methods, Phys. Rev. A 92, 033612 (2015).

[66] L. A. Peña Ardila and S. Giorgini, Bose polaron problem: Effect of mass imbalance on binding energy, Phys. Rev. A 94, 063640 (2016).

[67] F. Grusdt, G. E. Astrakharchik, and E. Demler, Bose polarons in ultracold atoms in one dimension: Beyond the Fröhlich paradigm, New J. Phys. 19, 103035 (2017).

[68] L. Parisi and S. Giorgini, Quantum Monte Carlo study of the Bose-polaron problem in a one-dimensional gas with contact interactions, Phys. Rev. A 95, 023619 (2017).

[69] E. Akaturk and B. Tanatar, Two-dimensional Bose polaron using diffusion Monte Carlo method, Int. J. Mod. Phys. B 33, 1950238 (2019).

[70] J. Vlietinck, W. Casteels, K. V. Houcke, J. Tempere, J. Ryckebusch, and J. T. Devreese, Diagrammatic Monte Carlo study of the acoustic and the Bose-Einstein condensate polaron, New J. Phys. 17, 033023 (2015).

[71] D. Delande and G. Orso, Mobility Edge for Cold Atoms in Laser Speckle Potentials, Phys. Rev. Lett. 113, 060601 (2014).

[72] M. Pasek, G. Orso, and D. Delande, Anderson Localization of Ultracold Atoms: Where is the Mobility Edge? Phys. Rev. Lett. 118, 170403 (2017).

[73] S. S. Kondov, W. R. McGehee, J. J. Zirbel, and B. DeMarco, Three-dimensional Anderson localization of ultracold matter, Science 334, 66 (2011).

[74] G. Semeghini, M. Landini, P. Castilho, S. Roy, G. Spagnolli, A. Trenkwalder, M. Fattori, M. Inguscio, and G. Modugno, Measurement of the mobility edge for 3D Anderson localization, Nat. Phys. 11, 554 (2015).

[75] N. Cherroret, T. Scoquart, and D. Delande, Coherent multiple scattering of out-of-equilibrium interact- ing Bose gases, Ann. Phys. (NY) 435, 168543 (2021).

[76] D. S. Petrov and F. Werner, Three-body recombination in heteronuclear mixtures at finite temperature, Phys. Rev. A 92, 022704 (2015).

[77] S. Häfner, J. Ulmanis, E. D. Kuhnle, Y. Wang, C. H. Greene, and $\mathrm{M}$. Weidemüller, Role of the intraspecies scattering length in the Efimov scenario with large mass difference, Phys. Rev. A 95, 062708 (2017).

[78] A. Ciamei, S. Finelli, M. Inguscio, A. Trenkwalder, and M. Zaccanti, Proceedings of the 52nd Annual Meeting of the APS Division of Atomic, Molecular and Optical Physics (APS, Ridge, 2021), Vol. 66.

[79] J. H. Bardarson, F. Pollmann, and J. E. Moore, Unbounded Growth of Entanglement in Models of Many-Body Localization, Phys. Rev. Lett. 109, 017202 (2012).

[80] M. Serbyn, Z. Papić, and D. A. Abanin, Universal Slow Growth of Entanglement in Interacting Strongly Disordered Systems, Phys. Rev. Lett. 110, 260601 (2013).

[81] K. Agarwal, E. Altman, E. Demler, S. Gopalakrishnan, D. A. Huse, and M. Knap, Rare-region effects and dynamics near the many-body localization transition, Ann. Phys. (Berlin) 529, 1600326 (2017).

[82] M. Schreiber, S. S. Hodgman, P. Bordia, H. P. Lüschen, M. H. Fischer, R. Vosk, E. Altman, U. Schneider, and I. Bloch, Observation of many-body localization of interacting fermions in a quasirandom optical lattice, Science 349, 842 (2015)

[83] S. Sun and S. Syzranov, Equivalence of interacting semimetals and low-density many-body systems to single-particle systems with quenched disorder, arXiv:2104.02720.

[84] A. D. McLachlan, A variational solution of the time-dependent Schrodinger equation, Mol. Phys. 8, 39 (1964).

[85] P. Kramer and M. Saraceno, in Geometry of the Time-Dependent Variational Principle in Quantum Mechanics, edited by J. Ehlers, K. Hepp, R. Kippenhahn, H. A. Weidenmüller, and J. Zittartz, Lecture Notes in Physics Vol. 140 (Springer, Berlin, 1981).

[86] A. G. Basile and V. Elser, Equations of motion for superfluids, Phys. Rev. E 51, 5688 (1995).

[87] J. Haegeman, J. I. Cirac, T. J. Osborne, I. Pižorn, H. Verschelde, and F. Verstraete, Time-Dependent Variational Principle for Quantum Lattices, Phys. Rev. Lett. 107, 070601 (2011). 